\title{
Coexistence of Attention-Based Facilitation and Inhibition in the Human Cortex
}

\author{
Per H. Ghatan,*,† J en Chuen Hsieh,*,‡ Karl M. Petersson,* Sharon Stone-Elander,*,§ and Martin Ingvar* \\ *Cognitive Neurophysiology, Department of Clinical Neuroscience and §Karolinska Pharmacy, Karol inska Hospital; †Division of \\ Rehabilitation Medicine, Danderyd Hospital, Stockholm, Sweden; and ¥l nstitute of Neuroscience, National Yang-Ming \\ University and Neuroanesthesia and Pain Unit, Veterans General Hospital-Taipei, Taiwan, Republic of China
}

Received J une 11, 1997

\begin{abstract}
A key function of attention is to select an appropriate subset of available information by facilitation of attended processes and/or inhibition of irrelevant processing. Functional imaging studies, using positron emission tomography, have during different experimental tasks revealed decreased neuronal activity in areas that process input from unattended sensory modalities. It has been hypothesized that these decreases reflect a selective inhibitory modulation of nonrelevant cortical processing. In this study we addressed this question using a continuous arithmetical task with and without concomitant disturbing auditory input (task-irrelevant speech). During the arithmetical task, irrelevant speech did not affect task-performance but yielded decreased activity in the auditory and midcingulate cortices and increased activity in the left posterior parietal cortex. This pattern of modulation is consistent with a top down inhibitory modulation of a nonattended input to the auditory cortex and a coexisting, attention-based facilitation of taskrelevant processing in higher order cortices. These findings suggest that task-related decreases in cortical activity may be of functional importance in the understanding of both attentional mechanisms and taskrelated information processing. 1998 Academic Press

Key Words: positron emission tomography; regional cerebral blood flow; attention; facilitation; inhibition; parietal cortex; auditory cortex; cognition.
\end{abstract}

\section{INTRODUCTION}

A selection of a limited subset of externally and internally generated information is an essential function of attention (Kinchla, 1992) (c.f. Niell et al., 1995). The mechanisms by which these selections are made are debated. Both direct facilitation of task relevant processing and inhibitory modulation of channels processing irrelevant stimuli have been suggested (Corbetta et al., 1991; Niell et al., 1995). Functional imaging studies with positron emission tomography (PET) focus primarily on task-induced increases of neuronal activity. However, many studies have reported decreased regional cerebral blood flow ( $\mathrm{rCBF}$ ) in areas processing input from unattended sensory modalities. Such findings have been reported for comparisons within a sensory modality (Drevets et al., 1995; Frith et al., 1991a; Zatorre et al., 1994) and between different sensory modal ities (Courtney et al., 1996; Ghatan et al., 1995). A novel task that requires the subject's full attention induces a more prominent rCBF decrease than that of a practiced task (J enkins et al., 1994). It has been hypothesized that such CBF decreases reflect a selective suppression of task-irrelevant information processing (Frith et al., 1991b; Ghatan et al., 1995). In this study, we challenged the idea of top down modulation of activity in cortical areas not involved in taskrelevant processing. Using a factorial design (Fig. 1), we investigated the influence of task-irrelevant speech on the processing of an internally generated arithmetical task. From previous studies we know that the arithmetical task alone leads to prominent bilateral rCBF decreases in the auditory cortices (Ingvar et al., 1997). Irrelevant speech (random words) was used as auditory input since passive listening to words increases the activity in the auditory cortices (Grasby et al., 1994). This design was expected to reveal a relative decrease of the rCBF response caused by task-irrelevant speech in the superior temporal gyri bilaterally when presented concomitantly with the arithmetical task, i.e., whether attention to an internally generated cognitive task modulates the activations that irrelevant speech alone elicits in the auditory cortices.

\section{MATERIALS AND METHODS}

\section{Subjects}

Six right-handed male volunteers (age 20-24, written informed consent) participated in the study, which was approved by the local Ethics and Radiation Safety committees at the Karolinska Hospital. All subjects 
were healthy, had no past history of psychiatric, neurologic, or other medical illnesses and were not on medication.

\section{PET Data Acquisition and Analysis}

Data were obtained using a Siemens-CTI ECAT Exact HR PET camera operated with the septa retracted to allow a high sensitivity 3D sampling mode, generating bolus triggered 60-s tracer uptake images. Thesubject's head was fixed in position for the duration of the scanning period with an individually molded plastic helmet (Bergström et al., 1981). Prior to the blood flow studies a transmission scan was performed that was later used for attenuation correction. The volunteers received a bolus injection of $\left[{ }^{15} \mathrm{O}\right]$ butanol (Berridge et al., 1990) (500 MBq) through a left forearm cannula. The PET-images were realigned, spatially normalized into a common stereotactic space (Talairach and Tournoux, 1988), 3D-Gaussian filtered (16 mm FWHM), proportionally scaled to account for global confounders, and analyzed with statistical parametric mapping (SPM 95 (Friston et al., 1995)). The resulting set of voxel values for each contrast constitutes a statistical parametric map of the statistic SPM (t) that was transformed to standard normal distributed parameters, SPM (Z), and threshol ded at 3.09 (omnibus significance $P \leq 0.001$ ). The activated regions were then characterized in terms of spatial extent $(k)$ and peakheight $(u)$ of local maxima. Differences detected by visual inspection in the arithmetical/interference task minus rest/interference compared to the arithmetical task minus rest, were tested using a masking approach, thereby yielding a more sensitive method of analysis (Fletcher et al., 1995b, 1995a). This was done by masking the interaction contrast [task $\times$ interference] with relevant contrasts (thresholding at $Z=2.33$ or omnibus significance $P \leq 0.01$ ), accepting clusters of volume $>10$ voxels and containing a local maxima with Z score $>2.56(P \leq 0.005)$ as significant [c.f. Fletcher, 1995a (59); Fletcher, 1995b (79)]

\section{Experimental Design}

The subjects were acquainted with the experimental environment during the initial testing. They were informed about the procedure and were then allowed to practice, both aloud and silently, the arithmetical task with and without task-irrelevant speech until they could count sequentially without disruption. During the scanning, instructions and the irrelevant speech were relayed through earphones. Each task was started $15 \mathrm{~s}$ before the radio-tracer injection.

In the arithmetical tasks, a seed number between 400 and 900 was given and the subjects were instructed to silently and without any "language related movements" sequentially subtract 7 from this number. When briefly tapped on the dorsum of the left arm, they were to say aloud the number reached. This reporting was prompted twice during the scanning period and once at the end of each scan. Their eyes were closed during all scans. Before each resting condition scan, the subject was instructed to not think of "anything in particular." In the resting condition with the irrel evant speech task they were to listen passively to the words given and to do no particular contemplation regarding the context or the meaning. The irrelevant speech paradigm (random words) consisted of common concrete nouns with high familiarity (Snodgrass and Vanderwart, 1980), presented at a frequency of 1 word every $3 \mathrm{~s}$. The design of the study is specified in Fig. 1. Each subject was scanned 12 times, 3 scans in each condition.

The subjective experience of difficulty in the arithmetical task was assessed with a visual analogue scale. Speed was estimated by the number of sequential subtractions performed between each prompted report. Accuracy of the number reported was related to the seed number and/or the previously reported number.

\section{RESULTS}

\section{Behavioral Data}

The procedures in the PET camera and the head fixation were tolerated well by all subjects. The subjects scored the arithmetical task as significantly more difficult when solved concomitantly with irrelevant speech ( $3.9 \pm 1.5$ vs $2.4 \pm 1.2 ; \mathrm{P}<0.05$; Wilcoxon Signed Rank test) but their performance was not significantly affected (accuracy, 2.4; range, 1- 3 vs 2.5; range, 1-3; and speed, $31.3 \pm 13.9$ vs $29.8 \pm 12.6$ subtractions/scan).

FIG. 1. There were 4 different scanning-conditions. Each of the 6 subjects was subjected 3 times to each of the 4 conditions. The 12 scans were performed in three blocks with the 4 different scan conditions in a randomized order within each block.

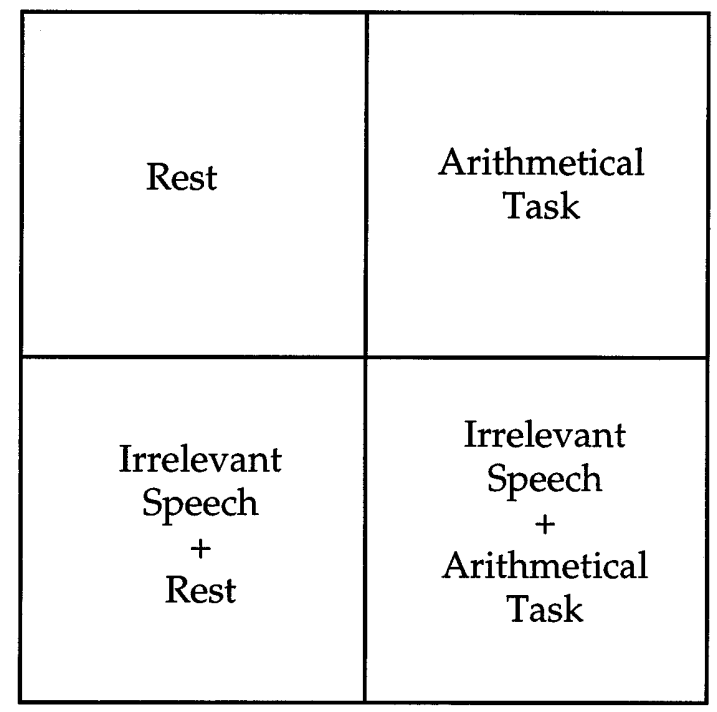




\section{Changes in rCBF}

The rCBF pattern elicited by the arithmetical task (arithmetical task-rest) was consistent with previously reported data (Ingvar et al., 1997) and showed bilateral activations of the parietal cortex, the prefrontal cortex, and the cerebellum. Activations were also found in the left premotor cortex and the left anterior cingulate cortex extending into the supplementary motor area. Bilateral decreases were evident in the medial prefrontal cortex and the medial and posterior temporal cortices including the auditory cortices. Irrelevant speech elicited, in the resting condition as well as during the arithmetical task, bilateral activations in the perisylvian cortex [superior temporal gyrus: left $Z=10.0$, $\mathrm{P}<0.001$; right $\mathrm{Z}=8.71 \mathrm{P}<0.001$ (corrected for multiple nonindependent comparisons)].

By visual inspection differences in the rCBF pattern between the arithmetical/interference task (arithmetical/interference task-rest/interference) and the arithmetical task (arithmetical task-rest) were found in the superior temporal lobes bilaterally, the left midcingulate region, the left anterior insular region (inhibitory modulation), and the left posterior parietal cortex (facilitatory modulation). Given the regional specific hypothesis (allowing the use of uncorrected $P$ values) addressing an inhibitory modulatory effect due to interference of the auditory cortex was confirmed in the interaction contrast (right BA 22/42, $[x, y, z]=[58,-30,4],, z=$ $3.09, P=0.001$, left $B A 22 / 42[x, y, z]=[-46,-34,0], Z=$ 2.86, $P=0.002$ ), see also Table $1(A)$ and Figs. 2 and 3. The facilitatory modulation in the posterior parietal cortex was confirmed in the interaction contrast showing a significant facilitatory effect in BA $7 / 40([x, y, z]=$ $[-26,-76,44], Z=4.03, P<0.05$, corrected for multiple nonindependent comparisons, since this region was not part of our original regional specific hypothesis), see also Table 1 (B) and Figs. 2 and 3. We also wanted to test the other differences (detected by visual inspection) in left supplementary motor area, left midcingulum, and left insula/frontal operculum. To maximize sensitivity we used a masking approach, masking the interaction contrast [ (rest with interference-arithmetical task with interference) - (rest-arithmetical task)] masked with [rest with interference-arithmetical task with interference] and thresholding at $Z=2.33$. This confirmed the inhibitory modulation of the left midcingulate cortex (BA 24), the left supplementary motor area (BA 6), the left anterior insula/frontal operculum (BA 14/49) (Table 2), and the left inferior frontal gyrus (BA 47).

\section{DISCUSSION}

Our findings are consistent with both facilitatory as well as inhibitory modulation of cortical activity when concomitant stimuli are experienced as disturbing for
TABLE 1

\begin{tabular}{lllll}
\hline & & & $P$ & $(X, Y$, \\
Region/structure & $B A$ & $Z$ & $\left(Z_{\max }>\mathrm{u}\right)$ & $Z \mathrm{~mm})$ \\
\hline
\end{tabular}

(A) Relative decreases induced by irrelevant speech during the arithmetical task

Right auditory cortex:

$\mathrm{vol}=146$

Superior temporal gyrus

BA22, BA42 $3.09 \quad 0.001 \quad 58-304$

Left auditory cortex: $\mathrm{vol}=58$

Superior temporal gyrus

BA42, BA22 $2.86 \quad 0.002 \quad-46-340$

(B) Relative increases induced by irrelevant speech during the arithmetical task

Left posterior parietal lobe: vol $=164$

Parietal lobe

BA $7 / 40$

4.03

0.047 $-26-7644$

Note. (A) Inhibitory modulatory effects were confirmed in the auditory cortex ( $P$ values uncorrected). (B) F acilitatory modulatory effects were seen in the posterior parietal region ( $P$ values corrected). Anatomical structures and Brodmann areas (BA) refer to the Talaraich-Tournoux atlas (Talairach and Tournoux, 1988) and the anatomical space of Greitz et al. (1991) and are given for the extension of the significant regions. BA, Brodmann area; vol, number of voxels in the cluster.

the attended task. Furthermore, these two modulatory effects coexist at different levels of cognitive processing. The disturbing effect of task-irrelevant speech on the arithmetical processing reported by the subjects had no effect on task-performance. This indicates that the arithmetical processing is left intact while attentionbased modulations due to the task irrelevant speech input are reflected in the rCBF changes detected in the interaction analysis.

The relative decreases in the auditory cortices may reflect a top down modulation of auditory processing that serves as a selective suppressor of task-irrelevant input (Niell et al., 1995). Electrophysiological data support such a mechanism of an early gain control based on attention in the auditory cortices (Hillyard et al., 1995). Increased demand on memory/attention in a nondisturbed working memory task also enhances the suppression of the auditory cortices (Grasby et al., 1994). Here, the demand on working memory in the arithmetical task was constant by design, but there was an increased demand for focused attention when the task was processed concomitantly with taskirrelevant speech. I rrelevant speech, while not in the focus of attention, might be automatically processed in the language system (Frith et al., 1995a) and thereby interfere with the arithmetical task. Deficiency of such a top down modulation in the superior temporal gyrus have been described in chronic schizophrenic patients (Frith et al., 1995b). Shulman et al. also addressed top 


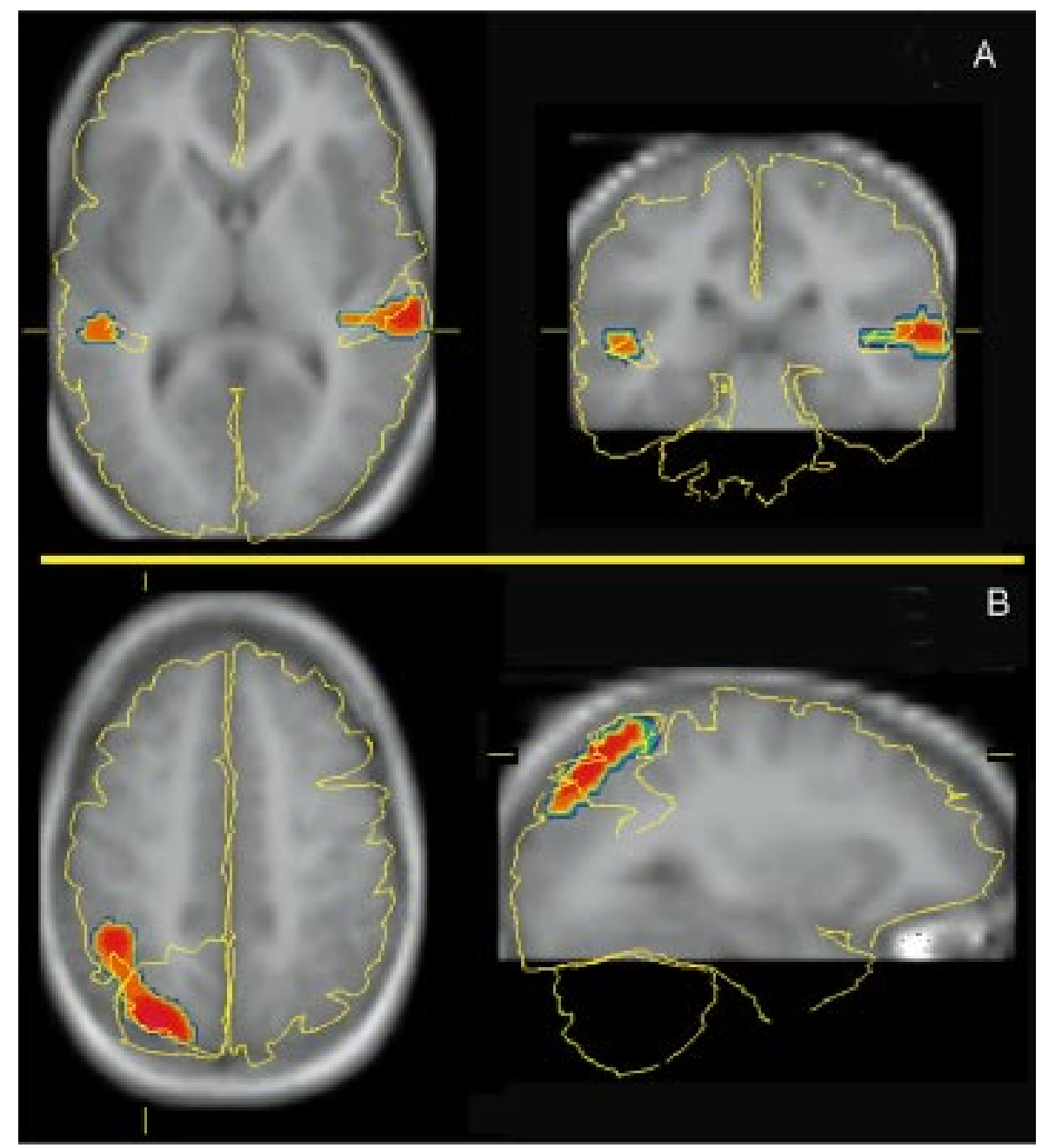

FIG. 2. A statistical parametric map (SPM) showing areas significantly modulated by irrelevant speech during the arithmetical task (interaction [interference $\times$ task]). The SPM (Z):s are displayed in the anatomical space of Greitz et al. (1991) and red denotes voxels with Z $\geq$ 2.33 or omnibus significance $\mathrm{P} \leq 0.01$. TherCBF data are superimposed on an average structural MRI image. (A) shows the bilateral decreases in the auditory cortex (superior temporal gyrus). BA 42 from the Greitz data base (Greitz et al., 1991) is denoted in green. The deactivations extend into BA 22, especially on the right side. The horizontal plane is at the level of $z=4$ and the coronal plane at $y=33$. (B) shows the increase in the left posterior parietal cortex. BA 7 is denoted in green. The activation extended into BA 40. The horizontal plane is at the level of $\mathrm{z}=44$ and the sagittal plane at $\mathrm{x}=30$.

down modulation of task irrelevant input in the auditory cortex in a post hoc analysis of nine previous studies involving visual discrimination (Shulman et al., 1997). Replicable decreases in the auditory cortices were inconsistent in their analysis within each study, but an analysis that combined data across studies yielded modest but significant decreases. The present study specifically addresses this question of a top down modulation of the auditory cortex. The use of an internally generated task allows the conclusion that the modulation is internally generated and thereby the result of a top-down mechanism and not a general cross-modal mechanism in an externally driven task (e.g., a visual task) (Haxby et al., 1994; Shulman et al., 1997). A related perspective on the inhibitory modula- tion of the auditory cortex conceptualizes this in terms of a failure of attentional selection in the arithmetical/ interference task as compared to rest/interference. In other words, it is possible that subjects attend to the irrel evant speech (actively sel ected) in the rest/interference condition, but did so to a lesser degree in the arithmetical/interference condition. On the other hand, a failure of attentional selection would not explain the need of a facilitatory modulation of task-relevant processing. The modulatory effect may be a combination of both selective filtering and a lack of attentional selection.

The finding of a relative decrease of $\mathrm{rCBF}$ in the auditory cortices is consistent with inhibitory modulation of processing prior to higher order categorization of 

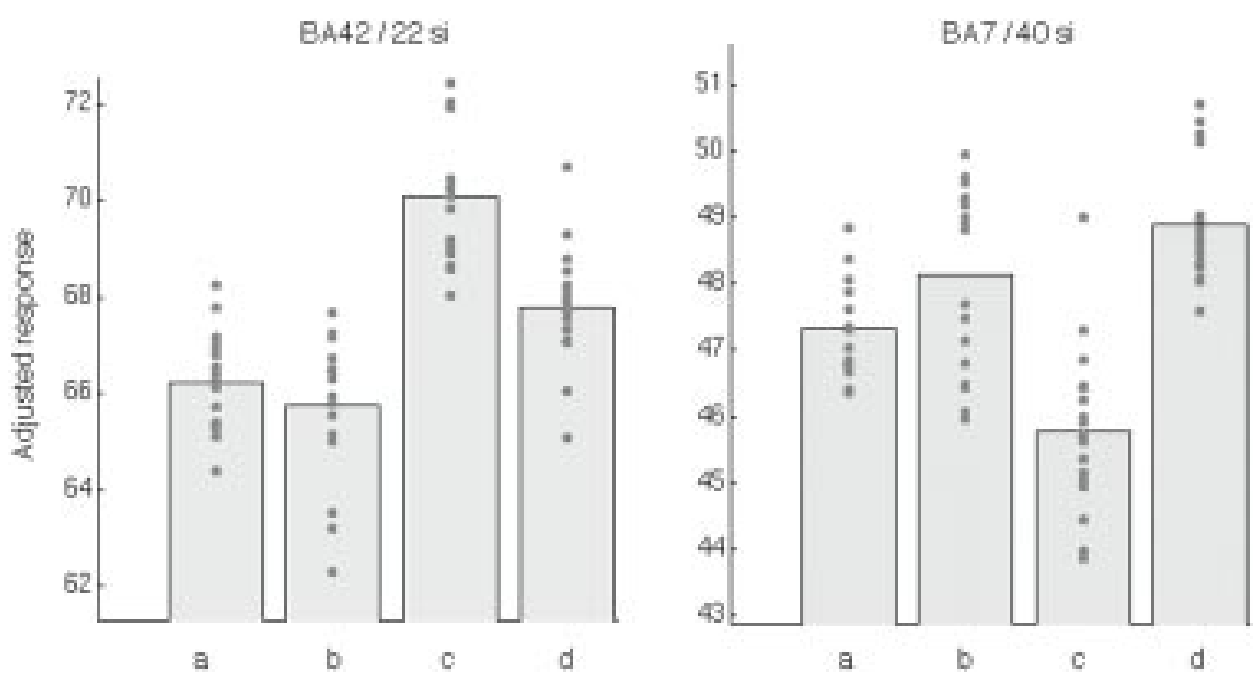

FIG. 3. Bar diagram showing the adjusted $\mathrm{rCBF}$ response for the respective experimental conditions in the left auditory cortex (BA 42/22) and the left posterior parietal cortex (BA 7/40). (a) rest; (b) arithmetical task; (c) rest + interference; (d) arithmetical task + interference.

the information content (Broadbent, 1958). On the behavioral level the information content in the taskirrelevant speech was filtered since, when questioned, the subjects could not recall the words following each scan (except one subject that recalled one word). The anterior part of the insula is connected with limbic, paralimbic, and autonomic structures and represents a polymodal convergence zone (Mesulam and Mufson, 1985). The decrease in the left anterior insular region as a result of irrelevant speech during the arithmetical task could reflect suppression of episodic encoding and

TABLE 2

\begin{tabular}{|c|c|c|c|c|}
\hline Region/structure & BA & Z & $\left(Z_{\max }^{P}>u\right)$ & $\begin{array}{l}(X, Y \\
Z m m)\end{array}$ \\
\hline \multicolumn{5}{|c|}{$\begin{array}{l}\text { Relative decreases induced by irrelevant speech during the } \\
\text { arithmetical task }\end{array}$} \\
\hline \multicolumn{5}{|l|}{$\begin{array}{l}\text { Left mid cingulate: } \\
\qquad \mathrm{vol}=59\end{array}$} \\
\hline $\begin{array}{l}\text { Cingulate gyrus } \\
\text { Left supplementary } \\
\text { motor cortex: } \\
\text { vol }=115\end{array}$ & BA24 & 2.96 & 0.002 & $-4-1440$ \\
\hline $\begin{array}{l}\text { Superior frontal gyrus } \\
\text { Left insula/frontal oper- }\end{array}$ & BA6 & 2.80 & 0.003 & $-14-2064$ \\
\hline $\begin{array}{l}\quad \text { culum: vol }=10 \\
\mathrm{vol}=15\end{array}$ & BA14/49 & 2.81 & 0.001 & -26264 \\
\hline Inferior frontal gyrus & BA47 & 2.74 & 0.003 & $-2420-20$ \\
\hline
\end{tabular}

Note. The results of testing the interaction contrast [(rest with interference - arithmetical task with interference) - (rest - arithmetical task)] in left supplementary motor area, left midcingulum, and left insula/frontal operculum (masking with [rest with interference - arithmetical task with interference], thresholding at $Z=2.33$ and accepting clusters of volume $>10$ voxels and containing a local maxima with $Z$ score $>2.56$ as significant). BA, Brodmann area; vol, number of voxels in the cluster. may explain the fact that the subjects did not remember the speech input. A possiblealternative explanation for the rCBF modulation in theanterior insula is that it could reflect an attention-based modulation of autonomic responses due to the experienced disturbance. There are extensive interconnections between the anterior insular region and the amygdala. The latter has been ascribed a role in the process of determining the affectivetone of experience and behavior (Mesulam and Mufson, 1985).

The left midcingulate region mediates appropriate motor and vocal responses and is selectively activated in tasks in which response selection is important, as for example, theStroop word color interferencetask (George et al., 1994). The arithmetical task used here contains no inherent conflict in the response selection. It is a single task with the demand to override the attention drift toward a potentially interfering auditory input. Corroborating our findings, a decreased activity in this part of the cingulate cortex has been correlated with decreases in superior temporal regions when the memory load was gradually increased (Grasby et al., 1994).

The left posterior parietal cortex is important for arithmetical processing (Ingvar et al., 1997) and lesions here are known to invoke acalculia (Henschen, 1919; Takayama et al., 1994). Its function has also been related to the phonological component of working memory (Baddeley, 1991; Paulesu et al., 1993; Petrides et al., 1993; Smith et al., 1996; Zatorre et al., 1992). Behavioral data show that irrelevant speech influences the phonological storein working memory tasks (Baddeley, 1991). Two previous PET studies have described a positive correlation between memory load and activity in the posterior parietal cortex (Grasby et al., 1994; 
Zatorre et al., 1996). We interpret the increased activity in this region during task-irrelevant speech and arithmetical processing as evidence for a facilitatory modulation of activity in a region necessary for processing the attended task (Niell et al., 1995).

Frith et al. have suggested that the inhibitory modulation of activity in the superior temporal region can be mediated by the dorsolateral prefrontal cortex (Frith et al., 1991b) and our findings do not contradict this. However, there are prominent cortico-cortical connections between the parietal cortex and the superior temporal gyrus, midcingulate cortex, and SMA (Cavada and Goldman-Rakic, 1989). It has been hypothesized that the posterior parietal cortex is a crucial component in the posterior network of attention (Mesulam, 1990; Posner, 1990); hence, we speculate that this region can also elicit a modulatory effect on the superior temporal region.

\section{CONCLUSION}

In conclusion, we have presented evidence that is consistent with the existence of an active modulatory attention mechanism by which task-irrelevant input in the auditory cortices is suppressed. These findings suggest that task-induced decreases of activity that have been reported in many functional imaging studies may be related to cortical mechanisms of attention. From a functional point of view, the ability to limit the cortical processing related to nonattended tasks offers a relevant mechanism for the reduction of information processing. Our data are also consistent with a coexisting facilitation of task-relevant processing, in our case, within the left parietal cortex, an area that is of primary importance for the attended task. These two modulatory effects, i.e., facilitatory and inhibitory, coexist at different levels of cognitive processing.

\section{ACKNOWLEDGMENTS}

We thank E. Andersson, M. Serrander, G. Printz, W. Pulka, G. Rosenquist, and P. Söderholm. This work was supported by grants from the Swedish Medical Research Council $(8276,9847)$, the Karolinska Institute, Swedish Bank Tercentenary F oundation, and the Knut and Alice Wallenberg F oundation.

\section{REFERENCES}

Baddeley, A. D. 1991. Working Memory. Clarendon Press, Oxford.

Bergström, M., Boëthius, J ., Eriksson, L., Greitz, T., Ribbe, T., and Widèn, L. 1981. Head fixation device for reproducible position alignment in transmission CT and positron emission tomography. J . Comp. Assist. Tomogr. 5:136-141.

Berridge, M., Cassidy, M., and Miraldy, F. 1990. A routine synthesis of ${ }^{15} \mathrm{O}$-labelled butanol for positron emission tomography. J . Nucl. Med. 31:1727-1731.

Broadbent, D. 1958. Perception and Communication. Pergamon Press, London.
Cavada, C., and Goldman-Rakic, P. S. 1989. Posterior parietal cortex in rhesus monkey: I. Parcellation of areas based on distinctive limbic and sensory corticocortical connections. J . Comp. Neurol. 287:393-421.

Corbetta, M., Miezen, F. M., Dobmeyer, S., Shulman, G. L., and Petersen, S. E. 1991. Selective and divided attention during visual discrimination of shape, color and speed: Functional anatomy by positron emission tomography. J Neurosci 11:2383-2402.

Courtney, S. M., Ungerleider, L. G., Keil, K., and Haxby, J . V. 1996. Object and spatial visual working memory activate separate neural systems in human cortex. Cereb. Cortex 6:39-49.

Drevets, W. C., Burton, H., Videen, T. O., Snyder, A. Z., Simpson, J . R., J r., and Raichle, M. E. 1995. Blood flow changes in human somatosensory cortex during anticipated stimulation. Nature 373: 249-252.

Fletcher, P. C., Frith, C., Grasby, P. M., Frackowiak, R. S. J ., and Dolan, R. J . 1995b. Brain systems for encoding and retrieval of auditory-verbal memory. Brain 118:401-416.

Fletcher, P. C., Frith, C. D., Baker, S. C., Shallice, T., Frackowiak, R. S. J ., and Dolan, R. J. 1995a. The mind's eye-precuenus activation in memory-related imagery. Neuroimage 2:195-200.

Friston, K. J ., Holmes, A. P., Worsley, K. J ., Poline, J .-P., and Frackowiak, R. S. J. 1995. Statistical parametric maps in functional imaging: A general linear approach. Hum. Brain Mapp. 2:189-210.

Frith, C., Kapur, N., Friston, K., Liddle, P., and Frackowiak, R. 1995a. Regional cerebral activity associated with the incidental processing of pseudowords. Hum. Brain Mapp. 3:153-160.

Frith, C. D., Friston, K., Liddle, P. F., and Frackowiak, R. S. J . 1991a. Willed action and the prefrontal cortex in man: A study with pet. Proc. R. Soc. Lond. B 244:241-246.

Frith, C. D., Friston, K. J ., Herold, S., Silbersweig, D., Fletcher, P., Cahill, C., Dolan, R. J ., Frackowiak, R. S., and Liddle, P. F. 1995b. Regional brain activity in chronic schizophrenic patients during the performance of a verbal fluency task. Br. J . Psychiatr. 167:343349.

Frith, C. D., Friston, K. J ., Liddle, P. F., and Frackowiak, R. S. J . 1991b. A Pet Study of word finding. Neuropsychology 29:11371148.

George, M. S., Ketter, T. A., Parekh, P. I., Rosinsky, N., Ring, H., Casey, B. J ., Trimble, M. R., Horwitz, B., Herscovitch, P., and Post, R. M. 1994. Regional brain activation when selecting a response despite interference: An H215O PET study of the Stroop and an emotional Stroop. Hum. Brain Mapp. 1:194-209.

Ghatan, P. H., Hsieh, J.-C., Wirsén-Meurling, A., Wredling, R., Eriksson, L., Stone-Elander, S., Levander, S., and Ingvar, M. 1995. Brain activation induced by the perceptual maze test: A PET study of cognitive performance. N euroimage 2:112-124.

Grasby, P. M., Frith, C. D., Friston, K.J ., Simpson, J ., Fletcher, P. C., Frackowiak, R. S., and Dolan, R. J . 1994. A graded task approach to the functional mapping of brain areas implicated in auditoryverbal memory. Brain 117:12711-11282.

Greitz, T., Bohm, C., Holte, S., and Eriksson, L. 1991. A computerized brain atlas: Construction, anatomical content and some applications. J . Comput. Assist. Tomogr. 15:26-38.

Haxby, J . V., Horwitz, B., Ungerleider, L. G., Maisog, J . M., Pietrini, P., and Grady, C. L. 1994. The functional organization of human extrastriate cortex: A PET-rCBF study of selective attention to faces and locations. J . Neurosci. 14:6336-6353.

Henschen, S. E. 1919. Clinical and anatomical contributions on brain pathology. Arch. Neurol. Psychiatry 13:226-249.

Hillyard, S. A., Mangun, G. R., Woldorff, M. G., and Luck, S. J . 1995. Neural systems mediating selective attention. In The Cognitive Neurosciences (M. S. Gazzaniga, Ed.), pp. 665-681. The MIT Press, London, England. 
Ingvar, M., Ghatan, P., Wirsén-Meurling, A., Risberg, J ., von Heijne, G., Stone-Elander, S., and Ingvar, D. 1997. Alcohol activates the cerebral reward system in man. J . Stud. Alc., in press.

J enkins, I. H., Brooks, D. J ., Nixon, P. D., Frackowiak, R. S., and Passingham, R. E. 1994. Motor sequence learning: A study with positron emission tomography. J . Neurosci. 14(6): 3775-3790.

Kinchla, R. 1992. Attention. Annu. Rev. Psychol. 43:711-742.

Mesulam, M. M. 1990. Large-scale neurocognitive networks and distributed processing for attention, language and memory. Ann. Neurol. 28:597-613.

Mesulam, M. M., and Mufson, E. F. 1985. The insula of Reil in man and monkey. Architectonics, connectivity and function. In Cereb. Cortex (A. Peters and E. G. J ones, Eds.), pp. 179-226. Plenum Press, New York.

Niell, T., Valdes, L., and Terry, K. 1995. Selective attention and inhibitory control of cognition. In Interference and inhibition in cognition, (F. Dempster and C. Brainerd Eds.), pp. 207-261. Academic Press, San Diego.

Paulesu, E., Frith, C. D., and Frackowiak, R. S. J . 1993. The neural correlates of the verbal component of working memory. Nature 362:342-345.

Petrides, M., Alivisatos, B., Meye, E., and Evans, A. C. 1993. Functional activation of the human frontal cortex during the performance of verbal working memory task. Proc. Natl. Acad. Sci. USA 90:878-882.
Posner, M. I. 1990. The attention system of the human brain. Annu. Rev. Neurosci 13:25-42.

Shulman, G. L., Corbetta, M., Buckner, R. L., Raichle, M. E., Fiez, J . A., Miezin, F. M., and Petersen, S. E. 1997. Top-down modulation of early sensory cortex. Cereb Cortex 7:193-206.

Smith, E. E., J onides, J ., and Koeppe, R. A. 1996. Dissociating verbal and spatial working memory using PET. Cereb. Cortex 6:11-20.

Snodgrass, J . G., and Vanderwart, M. J . 1980. A standardized set of 260 pictures: Norms for name agreement, image agreement, familiarity and visual complexity. J . Exp. Psychol. Hum. Learn. Memory 2:174-215.

Takayama, Y., Sugishita, M., Akiguchi, I., and Kimura, J . 1994. I solated acalculia due to left parietal lesion. Arch. Neurol. 51:286291.

Talairach, J ., and Tournoux, P. 1988. Co-Planar Stereotaxic Atlas of theHuman Brain. George Thieme Verlag, Stuttgart.

Zatorre, R. J ., Evans, A. C., and Meyer, E. 1994. Neural mechanisms underlying melodic perception and memory for pitch. J . Neurosci. 14:1908-1919.

Zatorre, R. J ., Evans, A. C., Meyer, E., and Gjedde, A. 1992. Lateralisation of phonetic and pitch discrimination in speech processing. Science 256:846-849.

Zatorre, R. J ., Meyer, E., Gjedde, A., and Evans, A. C. 1996. PET studies of phonetic processing of speech: Review, replication, and reanalysis. Cereb. Cortex 6:21-30. 